\title{
Lapurdum
}

Euskal ikerketen aldizkaria | Revue d'études basques |

Revista de estudios vascos | Basque studies review

$16 \mid 2012$

Numéro XVI

\section{ELEBIDUN : un projet de recherche sur l'apprentissage bilingue basque-français à l'école (primaire)}

Jean Casenave, Itziar Idiazabal, Ibon Manterola et Beñat Lascano

\section{(2) OpenEdition}

Journals

\section{Édition électronique}

URL : http://journals.openedition.org/lapurdum/2344

DOI : $10.4000 /$ lapurdum. 2344

ISSN : 1965-0655

Éditeur

IKER

Édition imprimée

Date de publication : 1 octobre 2012

Pagination : 15-25

ISSN : 1273-3830

\section{Référence électronique}

Jean Casenave, Itziar Idiazabal, Ibon Manterola et Beñat Lascano, «ELEBIDUN : un projet de recherche sur l'apprentissage bilingue basque-français à l'école (primaire) », Lapurdum [En ligne], 16 | 2012, mis en ligne le 03 décembre 2014, consulté le 01 mai 2019. URL : http:// journals.openedition.org/lapurdum/2344; DOI : 10.4000/lapurdum.2344 


\title{
ELEBIDUN: Un projet de recherche sur l'apprentissage bilingue basque-français à l'école (primaire)
}

\author{
Jean CASENAVE ${ }^{1}$ Itziar IDIAZABAL², Ibon MANTEROLA³ \\ Beñat LASCANO 4 \\ Université Michel de Montaigne-Bordeaux $3^{1}$, IKER (UMR 5478) ${ }^{1,4}$, Uni- \\ versité du Pays basque UPV/EHU2,3, ELEBILAB ${ }^{2,3}$, Université de Pau et des \\ Pays de l'Adour ${ }^{4}$
}

\section{Introduction $^{1}$}

Depuis plusieurs décennies, il existe une offre de scolarisation en langue basque dans les établissements scolaires du Pays basque nord (Pyrénées Atlantiques). Les élèves qui suivent une scolarité bilingue sont inscrits soit dans un établissement qui pratique la méthode dite immersive (Ikastolak de Seaska) soit dans un modèle à parité horaire dans un établissement public ou privé ${ }^{2}$. Ainsi, il est possible pour un élève de mener l'apprentissage de la langue basque en parallèle avec celui du français depuis l'école primaire jusqu'au lycée. Cette offre

1.- L'enquête sur le terrain a été menée et coordonnée par Elixabet Andueza, Julene Maia et Beñat Lascano. Nous remercions les élèves de CP, CE2 ET CM2 qui ont apporté leur concours enthousiaste à cette collecte. Nous exprimons également toute notre reconnaissance aux établissements et aux équipes enseignantes qui ont accepté de participer au projet ainsi qu'à la direction pédagogique de Seaska et aux inspections primaires de l'Education Nationale des circonscriptions d'Anglet et de Saint-Jean de Luz qui ont délivré les autorisations nécessaires.

Enfin, nous adressons tous nos remerciements au Conseil Général des Pyrénées Atlantiques qui a financé l'enquête sur le terrain et l'Office public de la langue basque, partenaire du projet qui a apporté une aide financière.

La partie de la recherche effectuée par ELEBILAB conte avec le support des projets FFI2012-37884-C03-01 du MINECO et IT676-13 du Gouvernement basque.

2.- Le modèle des Ikastolak tout comme celui des sections à parité horaire constituent des modèles immersifs pour les élèves scolarisés dans une langue autre que celle apprise à la maison (Idiazabal \& Dolz, 2013). En conséquence, le modèle en basque des Ikastolak est considéré comme immersif pour les enfants provenant de familles monolingues francophones, tandis que le modèle à parité horaire basque/français est lui partiellement immersif pour les enfants de première langue basque ou français. 
de scolarisation est en développement constant (38 ouvertures sur les 6 dernières années scolaires) et elle concerne aujourd'hui plus d'une école sur deux (58 \%), 34 \% des élèves du primaire et $43 \%$ des enfants en maternelle.

Affectée de façon particulièrement critique par l'affaiblissement de la transmission familiale, la population bascophone commence cependant à compenser cette perte par le développement d'une éducation bilingue. Le modèle d'immersion bascophone destiné aux enfants qui possèdent le français pour première langue comme celui des enfants qui ont le basque comme première langue favorisent l'émergence de générations bilingues. Lintérêt de ce phénomène est d'ordre socioculturel (processus de revitalisation d'une langue minoritaire et intégration sociale), individuel (bénéfices cognitifs, affectifs et identitaires) et scientifique. En effet cette recherche relève du domaine des Sciences du langage. Certaines disciplines de ce domaine comme la sociolinguistique, la psycholinguistique et la didactique des langues (pour sa partie centrée sur le bilinguisme et le plurilinguisme) sont directement concernées par la problématique étudiée à l'occasion du projet de recherche ELEBIDUN. C'est un sujet qui a un impact local mais aussi européen et plus largement, international dans le cadre de la recherche sur le plurilinguisme.

Malgré l'augmentation régulière des effectifs d'élèves scolarisés dans les cursus bilingues, ce type d'enseignement n'a pas encore donné lieu à un projet scientifique d'envergure dans la partie nord du Pays basque. Les études qui ont été faites jusqu'à présent ont été essentiellement d'ordre comptable et quantitatif en dehors de quelques articles du domaine sociolinguistique publiés par Jean-Baptiste Coyos (2006; 2007a ; 2007b ; 2008a ; 2008b) et de quelques analyses phonologiques et relatives au code switching (Epelde (2008, Epelde $\&$ Oyharçabal (à paraître). A titre d'exemple, on peut citer les statistiques publiées depuis sa création par l'Office public de la langue basque (nombre d'élèves scolarisés en basque ; nombre d'établissements offrant des filières immersives ou à parité horaire, etc. $)^{3}$.

Le projet de recherche intitulé ELEBIDUN, se situe dans une perspective de recherche d'ordre qualitatif. Il s'agit d'étudier les processus d'acquisition des langues basque et française par les élèves des sections bilingues soit en basque langue d'instruction principale (dite immersive) soit à parité horaire basque- et français. Ce développement des compétences linguistiques sera étudié de façon transversale tout au long de la scolarité primaire : CP, CE2, CM2.

A travers le projet ELEBIDUN, le laboratoire IKER (UMR 5478) s' $^{\prime}$ ouvre à la recherche sur l'acquisition des langues et le bilinguisme en milieu scolaire. Ce projet sera mené avec le laboratoire ELEBILAB ${ }^{5}$ (Laboratoire sur le bilinguisme, groupe de recherche HIJE (« Hizkuntzaren jabekuntza eta erabilerak-Acquisition et usages de langues ») de l'Université du Pays basque. Cette équipe a conduit de nombreuses recherches dans le domaine de l'apprentissage des langues et des problématiques du bilinguisme au cours des dernières années.

Le projet de recherche ELEBIDUN vient se greffer sur une recherche similaire que

3.- Office public de la langue basque : http://www.mintzaira.fr/

4.- IKER - UMR 5478: www.iker.cnrs.fr/ Unité mixte de recherche (UMR5478 Université Michel de Montaigne-Bordeaux 3 ; Université de Pau et des Pays de l'Adour ; Centre national de la recherche scientifique) centrée sur l'étude de la langue et les textes basques.

5.- ELEBILAB : www.elebilab.com/ 
le laboratoire ELEBILAB a développée à partir d'enquêtes effectuées dans la Communauté autonome basque et dans la Communauté forale de Navarre. La collecte du corpus, constitué par des productions orales et écrites effectuées par des élèves de 5-6 ans, 8-9 ans, 11-12 ans a été réalisée entre 2002 et 2010 et cette ligne de recherche a donné lieu à des nombreuses publications (Idiazabal \& Manterola, 2009 ; Idiazabal \& Dolz, 2013, Almgren et al., 2008 ; Manterola et al., 2013) ainsi qu'à la préparation d'une thèse doctorat qui a été soutenue en mars 2011 (Manterola, 2011).

La collaboration entre les deux laboratoires permettra donc, au terme de l'analyse des données recueillies dans les établissements des Pyrénées atlantiques, de créer un axe de recherche pour l'étude en profondeur des situations scolaires d'apprentissage bilingue (basque-français/ basque-espagnol) sur l'ensemble de l'aire bascophone actuelle.

\section{Objectifs et intérêt du projet}

\subsection{Intérêt du projet}

Comme indiqué en introduction, le projet de recherche ELEBIDUN met l'accent sur les aspects qualitatifs de l'apprentissage bilingue en milieu scolaire. Nous nous proposons d'étudier les processus d'acquisition et du développement des langues basque et française par les élèves des sections bilingues dans des contextes scolaires et sociolinguistiques contrastés (immersives et à parité horaire). Ce développement des compétences linguistiques sera étudié de façon transversale tout au long de la scolarité primaire : CP, CE2, CM2, suivant la même méthodologie de recherche que le groupe d'ELEBILAB.

Le fait d'utiliser la même méthodologie de recherche avec le même dispositif semiexpérimental et des élèves de groupes d'âge et de modèles bilingues similaires permet une comparaison des données particulièrement précise. Une étude récemment publiée peut être donnée comme exemple (Manterola et al., 2013). La production des connecteurs temporels en espagnol et basque y est étudiée chez des enfants de Ll espagnol scolarisés par immersion en basque L2. La recherche a pour but d'étudier le développement bilingue de ces enfants. Un autre groupe d'enfants bilingues de L1 basque est aussi pris en compte dans l'étude en tant que groupe de référence pour le développement du basque.

Lanalyse des connecteurs temporels se base sur la distinction entre des connecteurs ordinaires (puis, après, alors, etc.) qui se limitent à marquer une temporalité au niveau de la simple suite d'actions, et des connecteurs non-ordinaires (un jour, le jour suivant, tout d'un coup, quand, etc.) qui servent non seulement à identifier les phases et les épisodes narratifs mais aussi à distinguer l'arrière-plan des actions/événements.

Le graphique montre les données des deux groupes d'élèves de 5 et 8 ans en relation avec les pourcentages de textes contenant au moins un connecteur temporel non-ordinaire. 


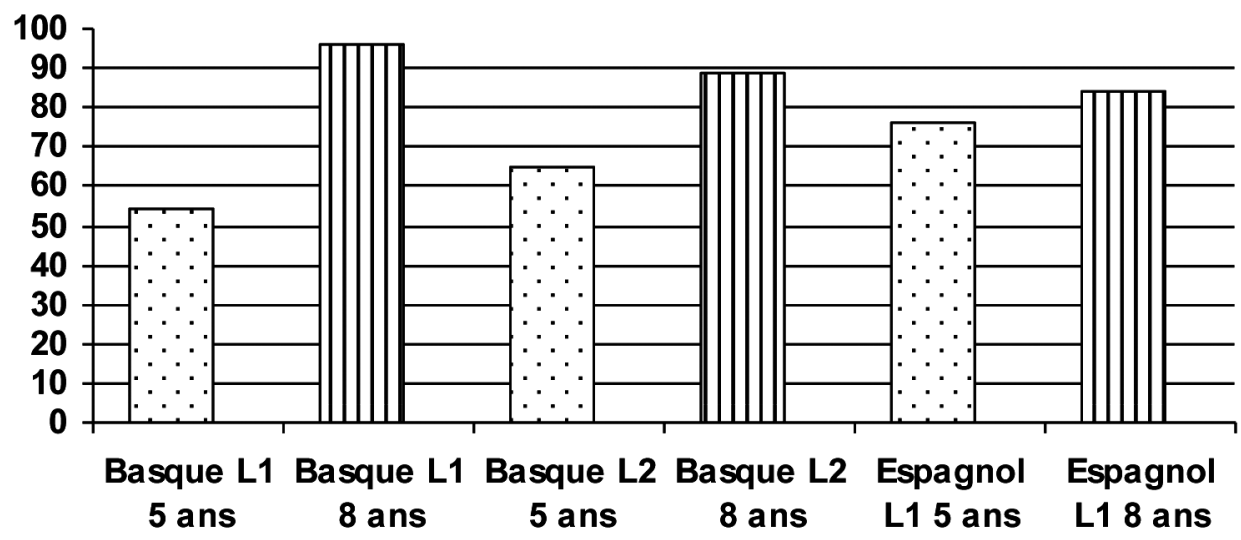

La production des connecteurs non-ordinaires augmente clairement avec l'âge, mais la différence est plus marquée en basque qu'en espagnol, indépendamment du fait que le basque soit L1 ou L2 pour l'enfant. En espagnol première langue (colonnes 5 et 6), par contre, même si les enfants utilisent très peu cette langue à l'école, la présence de ce type de connecteurs avec une valeur temporelle plus précise ou nuancée est déjà plus abondante à 5 ans. On peut donc considérer avec Berman \& Slobin (1994), De Weck (2000; 2005) que la présence de ce type de connecteurs dans les narrations constitue une caractéristique du développement langagier.

Une étude comparable sur les données des enfants bilingues du Pays basque Nord nous renseignera sur le développement du basque chez les deux groupes d'enfants. Elle pourrait aussi nous permettre de savoir si l'évolution des compétences linguistiques en français des enfants scolarisés dans les modèles d'immersion fait apparaître un retard par rapport à celle des monolingues francophones ou si elle suit la même progression.

Cette étude permettra de constituer un premier corpus de productions d'élèves scolarisés dans l'enseignement bilingue (langues basque et française) dispensé dans les établissements des Pyrénées Atlantiques. Son analyse mettra l'accent sur les compétences linguistiques acquises par les élèves inscrits dans une scolarité bilingue (langues basque et française).

Le corpus recueilli sera utilisé par les chercheurs qui travailleront sur le projet ELEBIDUN pour étudier les processus d'acquisition du basque et du français au cours de la scolarité primaire pour les locuteurs natifs comme pour les locuteurs formés par l'apprentissage scolaire. Cependant, ces résultats seront également intégrés aux bases de données constituées pour deux projets en cours dans le laboratoire IKER (UMR 5478), à savoir NORANTZ (Contact des langues et variation linguistique ; création d'un observatoire des nouveaux parlers bas- 
ques) et l'atlas syntaxique de la langue basque ${ }^{6}$.

\subsection{Objectifs :}

Les capacités linguistiques des élèves seront analysées dans un domaine de compétences préalablement bien établi. L'étude sera centrée sur le degré de maîtrise du discours narratif qui mobilise une bonne partie des outils linguistiques dont disposent les enfants du niveau primaire. Aussi, les résultats obtenus donneront une indication pertinente à propos de leurs capacités d'expression dans les deux langues.

De plus, en procédant à une étude transversale (CP, CE2, CM2), il sera possible d'obtenir une représentation des compétences linguistiques acquises (dans le domaine étudié) par les élèves bilingues durant toute leur scolarité primaire.

Il sera également intéressant d'observer le bénéfice que peuvent tirer d'une scolarisation bilingue aussi bien les élèves issus d'un milieu majoritairement bascophone (basque L1) que les élèves venant d'un milieu non bascophone (basque L2). En effet la compétence bilingue se révèle positive tant du point de vue cognitif et individuel, que du point de vue social et affectif. Il a été prouvé par exemple, que les élèves bilingues sont en capacité d'apprendre très facilement une troisième langue (Cenoz, 1996). Ils font preuve d'une flexibilité cognitive supérieure à leurs camarades unilingues (Baker, 2006) et montrent aussi des capacités métalinguistiques plus développées (Cummins, 1983). Du point de vue social et affectif, la connaissance de l'autre langue de la communauté, le basque en l'occurrence, permet de se sentir mieux intégré dans cette communauté. Le locuteur a le sentiment de participer à la revitalisation d'une langue minorée et au maintien de la diversité linguistique. Il prend part à une initiative sociale et culturelle aux fortes valeurs formatives et identitaires (Urla, 2012).

\subsubsection{Objectifs spécifiques en relation avec l'enseignement bilingue}

Concernant l'apprentissage du basque, il sera possible d'étudier la progression dans les domaines de la maîtrise de la morphosyntaxe, du lexique et des capacités discursives associées aux textes narratifs oraux parmi les élèves de basque Ll et chez leurs homologues de basque L2.

Il sera également intéressant de comparer les observations relatives aux acquisitions en langue basque réalisées dans nos établissements des Pyrénées atlantiques avec les résultats relevés par le laboratoire ELEBILAB dans la Communauté autonome basque et dans la Communauté forale de Navarre. La comparaison des résultats pourra donner des indications sur la situation d'ensemble dans l'aire bascophone.

La présence des pratiques dialectales pourra également être étudiée. De même, il sera possible pour la première fois d'observer les éléments de langue commune qui sont transmis par le biais de la scolarisation en langue basque. Ces observations seront mises en relation et en commun avec celles qui sont recueillies dans le cadre de deux autres projets de recherche menés à IKER (Norantz/Observatoire des parlers nouveaux; Atlas syntaxique).

En ce qui concerne les données relatives à l'acquisition du français, elles pourront être 
comparées avec celles qui résultent de l'enquête menée auprès des élèves unilingues (scolarisés uniquement en françaisqui ont été publiées à la suite d'études similaires qui ont été effectuées en France (pour des enfants monolingues et bilingues) ou dans d'autres pays francophones (Hickmann et al., 1995 ; De Weck, 1991).

Il faudra s'intéresser également aux stratégies métalinguistiques développées par des enfants bilingues, aux transferts linguistiques entre le français et le basque et vice-versa, et aussi aux mécanismes d'interlangue et de code switching entre les deux langues. Ces questions constituent des objets de recherche bien développés qui peuvent néanmoins s'enrichir avec l'étude ici proposée étant donné par exemple la distance linguistique et sociolinguistique entre les deux langues objets d'apprentissage (Meisel, 2007 ; Fayol, 1991).

Au niveau de la didactique bilingue les apports de cette recherche sont également importants : les mécanismes/recours de traduction, de comparaison, de contraste, de reformulation, de coordination ou complémentation entre les apprentissages des deux langues sont des objets d'étude très demandés d'après les exigences de formation bi-plurilingue actuelles (Conseil d'Europe, 2001). On peut étudier les données des élèves et des enseignants et il serait intéressant de comparer ces données avec celles d'autres recherches contrastées (Genesse, 2006 ; Gajo, 1999 ; Dolz \& Gagnon, 2008).

Enfin il y a aussi des objectifs généraux en relation avec le domaine des Sciences du langage. En tenant compte des sujets théoriques déjà abordés par les laboratoires partenaires (sémiotique narrative, organisation du texte/discours narratif) certains centres d'intérêt seront privilégiés (éléments de cohésion du discours narratif, relatifs notamment à l'emploi des verbes). Ainsi, il sera possible d'avancer sur des questions inexplorées à ce jour dans le domaine basque par la collaboration entre les deux laboratoires.

Nous considérons que les observations qui résulteront des objectifs définis ci-dessus constitueront un premier ensemble de données sur les acquis réalisés par les élèves inscrits dans une scolarité bilingue. Cette étude pourrait ultérieurement servir de base à d'autres projets de recherche menés conjointement par les deux laboratoires sur d'autres aspects de la scolarisation dans les systèmes bilingues.

Il sera aussi possible de travailler avec d'autres laboratoires qui étudient les questions liées à l'apprentissage de la L2 en général mais aussi en relation avec des situations plus spécifiques : la scolarisation des enfants issus des populations nouvellement arrivées (Akinci, 2001) ou la scolarisation des élèves dans les diverses langues régionales en France et en Europe.

\subsection{Résultats attendus}

Le projet de recherche ELEBIDUN permettra de constituer le premier corpus d'envergure produit par des élèves inscrits dans l'enseignement bilingue basque-français. Il donnera lieu, à partir des résultats de cette enquête, à la première recherche scientifique dans le domaine du bilinguisme (langues basque et française) tel qu'il est pratiqué dans la partie nord du Pays basque. Ce projet offrira également une description linguistique et psycholinguistique précise de l'usage des langues basque et française parmi les élèves de niveau primaire. En complétant les données fournies par le laboratoire ELEBILAB, il contribuera à constituer une vision d'ensemble de la situation dans toute l'aire bascophone. Dans la perspective de la recherche sur l'enseignement bilingue, le projet ELEBIDUN peut fournir une ressource didac- 
tique utilisable pour l'enseignement du basque. Il apportera aussi des observations inédites sur la question des interférences et des transferts linguistiques entre le basque et le français. Et bien évidemment, ELEBIDUN donnera des indications intéressantes sur l'apprentissage d'une L2, soit basque L2 pour des élèves de langue première française, soit français L2 pour des élèves de langue première basque.

\section{Méthodologie utilisée}

\subsection{Déroulement de la recherche}

La recherche sera divisée en deux phases : la collecte des données et leur analyse. Deux sortes de productions seront recueillies : textes oraux pour les élèves des trois niveaux étudiés, textes écrits pour les plus âgés. La collecte sera effectuée dans les deux langues (basque et français). Ensuite, les productions orales feront l'objet d'une transcription.

Pour ce qui concerne l'analyse, l'équipe chargée de la collecte établira une transcription des enregistrements. Le corpus ainsi constitué sera analysé selon un protocole scientifique défini par les membres des laboratoires IKER et ELEBILAB.

\subsection{La population étudiée}

Trois classes d'âge seront étudiées : CP, CE2, CM2, c'est-à-dire 6/7, 8/9 et 10/11 ans. La recherche concerne deux types d'établissements : des établissements offrant un enseignement en basque à temps complet (Ikastolak de Seaska) : enseignement immersif pour les enfants de Ll française et enseignement de maintien pour les enfants de L1 basque. Lautre type d'établissement fourni un enseignement à parité horaire, en immersion partielle. Le groupe monolingue sera sélectionné soit dans un établissement bilingue ou monolingue.

La population bilingue étudiée appartient donc à quatre types d'itinéraire bilingue, caractérisés selon les critères suivants : la première langue ; l'environnement sociolinguistique ; le système de scolarisation suivi. Le premier se réfère à des élèves issus d'une famille bascophone et qui évoluent dans un milieu bascophone avec une scolarité suivie en basque. Le deuxième itinéraire est celui des élèves issus d'une famille non bascophone qui vivent dans un entourage où la présence du basque est très faible et qui ont appris le basque par le biais de l'école immersive en basque. Ensuite il y a des élèves de familles bascophones qui habitent dans un milieu bascophone et qui sont scolarisés en immersion française partielle. Et le quatrième itinéraire concerne des élèves scolarisés aussi en immersion partielle en basque mais qui proviennent de familles et de milieux francophones.

En tant que groupe de contrôle nous réaliserons la même enquête avec un groupe monolingue francophone constitué des mêmes niveaux scolaires. 
Tableau récapitulatif des groupes d'élèves

\begin{tabular}{|c|c|c|c|c|}
\hline $\begin{array}{l}\text { Groupe } \\
\text { d'élèves }\end{array}$ & Classe & $\begin{array}{l}\text { Première } \\
\text { langue }\end{array}$ & $\begin{array}{l}\text { Environnement } \\
\text { sociolinguistique }\end{array}$ & $\begin{array}{l}\text { Système de } \\
\text { scolarisation }\end{array}$ \\
\hline Gl & $\begin{array}{c}\mathrm{CP} \\
\mathrm{CE} 1 \\
\mathrm{CM} 2\end{array}$ & Basque & Bascophone & Maintien \\
\hline G2 & $\begin{array}{c}\mathrm{CP} \\
\mathrm{CE} 1 \\
\mathrm{CM} 2\end{array}$ & Français & Francophone & Immersif \\
\hline G3 & $\begin{array}{c}\mathrm{CP} \\
\mathrm{CE} 1 \\
\mathrm{CM} 2\end{array}$ & Basque & Bascophone & À parité horaire \\
\hline G4 & $\begin{array}{c}\mathrm{CP} \\
\mathrm{CE} 1 \\
\mathrm{CM} 2\end{array}$ & Français & Francophone & À parité horaire \\
\hline G5 & $\begin{array}{c}\mathrm{CP} \\
\mathrm{CE} 1 \\
\mathrm{CM} 2\end{array}$ & Français & Francophone & Monolingue en français \\
\hline
\end{tabular}

\subsection{Dispositif pour la récolte des données}

Les capacités linguistiques des élèves en basque et en français seront étudiées à partir de la compétence narrative, en s'appuyant sur leur pratique du discours narratif. Il leur sera demandé de restituer à l'oral, individuellement et pour un interlocuteur désigné un récit qu'ils auront préalablement entendu de la bouche d'un adulte. Il s'agit d'un dispositif identique à celui qui a été utilisé par le groupe partenaire d'ELEBILAB.

Le choix du texte-discours narratif est lié au fait que le marqueur « genre » et les textes génériques sont des unités d'analyse pertinentes pour mesurer tout un ensemble de compétences linguistiques (Bronckart, 1996). Le genre narratif, et plus particulièrement le conte constitue ainsi un genre très utilisée dans le domaine de l'acquisition du langage monolingue et bilingue (Berman \& Slobin, 1994 ; Berman, 2009 ; Hickmann, 2001 ; Jisa, 2000 ; Severing $\&$ Verhoeven, 2001), ainsi que dans la didactique des langues (Beloki \& Manterola, 2007 ; Dolz \& Gagnon, 2008 ; Rosat, 1998).

Deux sortes de productions seront recueillies dans les écoles : des productions orales réalisées par les élèves des trois niveaux scolaires sélectionnés (CP, CE2, CM2); des productions écrites réalisées par les élèves les plus grands (CE2, CM2).

Les productions orales seront enregistrées puis transcrites pour constituer le corpus à analyser. Pour les plus grands (CM2 et éventuellement CE2 selon les moyens disponibles), une production écrite sera également relevée.

Les productions orales seront recueillies dans des classes entières afin de ne pas perturber les conditions de la collecte. La sélection des productions qui seront transcrites se fera ultérieurement en fonction des deux profils d'élèves qui ont été choisis (élèves issus majoritai- 
rement d'un milieu bascophone ; élèves issus majoritairement d'un milieu non bascophone). Des ensembles de 12 à 15 unités seront établis de façon à constituer des corpus cohérents et probants.

\subsection{Les grilles d'analyse}

Les grilles d'analyse seront définies ultérieurement en accord avec les membres du laboratoire ELEBILAB qui participent à l'étude selon les principes de la sémiotique narrative. La méthodologie adoptée prendra en compte les situations sociolinguistiques (pratiques de la langue basque ; dialectes, etc.) et pédagogiques (systèmes d'enseignement bilingue existant dans les P.A) qui configurent le terrain de l'enquête.

Les grilles d'analyse peuvent intégrer les apports du laboratoire ELEBILAB qui a déjà développé divers éléments d'analyse dans son corpus bilingue basque-espagnol, par exemple, l'autonomie narrative, l'organisation des contenus, la connexion, la cohésion nominale et verbale et la prise en charge énonciative (Manterola \& Almgren, 2013). La perspective interlinguistique entre le basque et le français peut constituer un point d'intérêt spécial dans le projet ELEBIDUN et peut ainsi donner lieu à des analyses comparatives telles que la production des verbes dans les contes. Des études liées à ce sujet montrent que les systèmes verbaux de deux langues fonctionnent de manière différente dans les textes narratifs notamment en ce qui concerne la production des marqueurs aspectuels, perfectifs et imperfectifs en lien avec les phases narratives (Bonnotte et al., 1993). Lacquisition de ces traits linguistiques spécifiques à chaque langue constituera donc un point d'étude parmi d'autres dans le cadre du projet ELEBIDUN.

\section{Bibliographie}

Akinci, M. A. (2001), Développement des compétences narratives des enfants bilingues turc-français en France âgés de 5 à 10 ans. München : Lincom Studies in Language Acquisition 03.

Almgren, M., Beloki, L., Idiazabal, I.\& Manterola, I. (2008), "Acquisition of Basque in successive bilingualism: data from oral storytelling”, in P. Siemund, \& N. Kintana (ed.) Language Contact and Contact Languages. Amsterdam : John Benjamins.

Baker, C. (2006), Foundations of Bilingual Educations and Bilingualism, Clevedon: Multilingual Matters.

Beloki L. \& Manterola, I. (2007), "Ahozko Ipuin- Kontaketa Haur- Hezkuntzan: Ikerketa Esperimentaletik Didaktikara”In I. Idiazabal \& I. Garcia Azkoaga (Eds.) Ahozko hizkuntza. Euskararen azterketarako eta didaktikarako zenbait lan. (Sareko argitarapena, UPV-EHU) ISBN: 978-84-690-8853-1.

Berman, R. \& Slobin, D. (1994), Relating events in narrative : a crosslinguistic developmental study, Hillsdale, New Jersey, Lawrence Erlbaum.

Berman, R.A. (2009), «Language development in narrative contexts », in The Cambridge Handbook of Child Language, Ed. E.L. Bavin, Cambridge, CUP.

Bonnotte, I., Kaifer, A., Fayol, M. and Idiazabal, I. (1993), "Rôle des types de procès et du co- 
texte dans l'emploi des formes verbales de la narration." Langue Française 97, 81-101.

Bronckart, J.-P.1996, Activité langagière, textes et discours. Pour un interactionnisme socio discursif, Paris, Delachaux et Niestlé.

Bronckart, J.-P., 2007, Desarrollo del lenguaje y didáctica de las lenguas, Santa Fe, Miño Dávila.

Cenoz, J. (1998), "Multilingual Education in the Basque Country", in J. Cenoz \& F. Genesee (Ed.) Beyond Bilingualism: Multilingualism and Multilingual Education. Clevedon : Multilingual Matters.

Coyos, J.-B., (2005b)'Ecart entre connaissance et usage d'une langue minoritaire : essai de typologie des facteurs. Le cas de la langue basque, Politique linguistique et enseignement des Langues de France', Colloque organisé par l'Association Universitaire des Langues de France, Toulouse (France), 26-27/05/2005.

Coyos, J.-B., (2005c), "L'enseignement suffit-il à « sauver» une langue menacée ? Lexemple du Pays Basque », Lapurdum 10.

Coyos, J.-B. (à paraître), 'Aldaera eta aldaketa euskararen ezagutzan eta erabileran Ipar Euskal Herrian - Inkesta soziolinguistikoen aztertze kritikoa [Variation et changement dans la connaissance et l'usage de la langue basque au Pays Basque Nord - Examen critique des enquêtes sociolinguistiques]', Colloque Internationale Changements, variations et variantes dans la langue et les textes basques, 12-13 décembre 2008, Centre de recherche sur la langue et les textes basques Iker, Bayonne. Lapurdum XIII.

Cummins, J. (1983) "Interdependencia lingüística y desarrollo educativo de los niños bilingües”, Infancia y Aprendizaje, 1983, 21, 37-61.

De Weck, G. (1991), La cohésion dans les textes d'enfants. Paris: Delachaux et Niestlé.

De Weck, G. (2000), "Acquisition des capacités langagières”, in De Weck, G., Gajo, L., Moderato, P. \& Blanc-Perroto, Attraper le français. Acquisizione del linguaggio e bilinguism. Aoste: IRRSAE, Vallé D'Aoste.

De Weck, G. (2005), "Lappropriation des discours par les jeunes enfants” , in B. Piérart (ed.), Le langage de l'enfant: comment l'évaluer? Bruxelles: De Boeck.

Dolz, J. \& Gagnon, R. (2008), "Le genre du texte, un outil didactique pour développer le langage oral et écrit", Pratiques, 137-138, La didactique du français: hommages à Jean-François Halté. 179-198.

Epelde, I., (2008) 'Impacto fonológico del contacto entre lenguas: francés y vasco en contacto', Communication présentée l'Université d'Almeria (Espagne), le 3 avril 2008 (XXVI. Congrès de l'Asociación Espanola de Linguitica Aplicada-AESLA).

Epelde, I. \& Oyharçabal, B. (à paraître) "Code swithching en las variedades orientales del vasco", in Frans Gregeren \& Jefferey Company (édit.), Proceedings of 27th AESLA International Conference, Ciudad Real (26-28 mars 2009), à paraître dans un numéro spécial de RESLA (Revista Española de Lingüistica Aplicada).

Fayol, M. (1991), "Stories. A Psycholinguistic and Ontogenetic Approach to the Acquisition of Narrative Abilities" in G. Piéraut-Le Bonniec \& M. Dolitsky (ed.), Language Bases... Discourse Bases. Amsterdam: John Benjamins. 
Gajo, L. (1999), Enseignement des langues par immersion et activité métalinguistique. Enjeux didactiques, interactionnels et sociopolitiques. Université de Lausanne.

Genesee, F. (2006), "Una revisió dels programmes d'immersió en francès al canadà", Quaderns d'avaluació, 6. 56-77.

Hickmann, M. (2001), "Le développement de la cohésion discursive: le récit oral à l'oral chez l'enfant", Langage \& pratiques, n. 27, Langage oral: aspects developpementaux. 43-52.

Hickmann, M., Kail, M. \& Roland, F. (1995), "Cohesive anaphoric relations in French children's narratives as a fonction of mutual knowledge", First Language, 15. 277-300.

Idiazabal, I. \& Dolz, I. (2013), "Introducción: Diversidad lingüística y formación plurilingüe" in J. Dolz \& I. Idiazabal (eds.) Enseñar (lenguas) en contextos plurilingües. Bilbao: Servicio editorial de la Universidad del País Vasco /Euskal Herriko Unibertsitatea, 11-32.

Idiazabal, I. \& Manterola, I. (2009), "Euskal eredu elebidunak, murgilketa eta hizkuntzen irakaskuntza bateratua: kontzeptuen berrikusketa”, Euskera, 54, 2-1. 463-504.

Jisa, H. (2000), "Increasing cohesion in narratives : A developmental study of maintaining and introducing subjects in French", Linguistics, 38, 591-620.

Manterola, I. (2011) Euskarazko murgilketa aur hezkuntzan: Euskara-gaztelania Elebitasunaren azterketa. Ahozko ipuinen analisi diskurtsiboa eta didaktikoa, Doktorego tesia. UPV/EHU Argitarapen Zerbitzua.

Manterola, I. \& Almgren, M. (2013), "Las grandes líneas de progresión de los aprendizajes lingüísticos en la escolarización en euskera", in J. Dolz \& I. Idiazabal (eds.) Enseñar (lenguas) en contextos plurilingües. Bilbao: Servicio editorial de la Universidad del País Vasco /Euskal Herriko Unibertsitatea.

Manterola, I., Almgren, M. \& Idiazabal, I. (2013), "Basque L2 development in immersion school settings", International Journal of Bilingualism, 17(3) 375-391.

Manterola, I., Almgren, M. \& Idiazabal, I. (2013), «L'usage chez des enfants bilingues de connecteurs dans les contes oraux en basque L1 et L2 », in R. Delamotte \& M.A. Akinci (Eds.), Récits d'enfants. Développement, genre, contexte. Rouen : PURH.

Meisel, J. (2007), « Child second language acquisition or successive first language acquisition? », Arbeiten zur Mehrsprachigkeit- Working Papers in Multilingualism, Hamburg, Sonderforschungsbereich Mehrsprachigkeit, 33-64.

Rosat, M.C. (1998), "Comparaison des stratégies discursives d'étayage dans un conte et un récit d'expériences oraux", Travaux Neuchatelois de linguistique, 29. 29-47.

Severing, R. \& Verhoeven, L. (2001), "Bilingual narrative development in Papiamento abd Dutch", in L. Verhoeven \& S. Stromqvist (ed.) (2001), Narrative Development in a Multilingual Context. Amsterdam: John Benjamins.

Urla, J. (2012) Reclaiming Basque: Language, Nation, and Cultural Activism Reno: University of Nevada Press 